\title{
Three-Wave Longitudinal Survey on the Relationship between Neuroticism and Depressive Symptoms of First-Year College Students: Addictive Use of Social Media as a Moderated Mediator
}

\author{
Weiqi Mu ${ }^{1,2,+}$, Dongyun Zhu ${ }^{1,2,+}$, Yanhong Wang ${ }^{3}$, Fugui Li ${ }^{1,2}$, Liyuan Ye ${ }^{1,2}$, Kexin Wang ${ }^{4}$ and \\ Mingjie Zhou ${ }^{1,2, *(D)}$ \\ 1 CAS Key Laboratory of Mental Health, Institute of Psychology, Chinese Academy of Sciences, Beijing 100101, \\ China; muwq@psych.ac.cn (W.M.); aschenpeter@126.com (D.Z.); lifg@psych.ac.cn (F.L.); \\ yeliyuan06@gmail.com (L.Y.) \\ 2 Department of Psychology, University of Chinese Academy of Sciences, Beijing 100049, China \\ 3 Mental Health Counseling Center, Yang' En University, 362014 Quanzhou, China; wangyh@yeu.edu.cn \\ 4 College of Media and International Culture, Zhejiang University, Hangzhou 310007, China; \\ wangkexin0809@zju.edu.cn \\ * Correspondence: zhoumj@psych.ac.cn \\ + These authors contributed equally to this work.
}

Received: 30 June 2020; Accepted: 18 August 2020; Published: 20 August 2020

\begin{abstract}
First-year college students' adaptation problems and related mental health have attracted researchers' attention. The current research focuses on the depressive symptoms of first-year college students and aims to explore the relationship between the neuroticism trait and depressive symptoms, the mediating effect of addictive use of social media, and the moderating effect of psychological resilience. Three-wave longitudinal data from 1128 first-year students at a university in Fujian Province, China, were collected within three months of their enrollment. PROCESS macro for SPSS with bootstrapping was used to test the model. Results showed that the prevalence of moderate to severe severity of depressive symptoms in first-year students was $10.28 \%$ (T1) and $11.17 \%$ (T3). Addictive use of social media (T2) plays a moderated mediator role in the relationship between neuroticism (T1) and depressive symptoms (T3) of first-year students. Specifically, a low neuroticism individual does not necessarily have a less addictive use of social media. Psychological resilience (T1) moderated the above mediation. Implications for research and practice are discussed.
\end{abstract}

Keywords: depressive symptoms; neuroticism; addictive use of social media; psychological resilience; moderated mediation

\section{Introduction}

While college presents many students with opportunities for personal growth and enhancement, some students find the demands of college adjustment exceed their coping resources [1]. New college students begin their transition from adolescence to adulthood, and many of them have difficulty adapting to college life. The worse the adaptation of first-year students, the higher the risk of psychological disturbance [2,3]. Previous studies have shown that there is a significant correlation between depression symptoms and suicide ideation of college students $[4,5]$. Therefore, the mental health of first-year college students, especially depressive symptoms, deserves our attention. Many researches have demonstrated the closing association between personality traits and depression, especially the role of high neuroticism in developing depression [6-9]. Meanwhile, addictive use of social media has become an area of increasing research interest. A large national survey in Norway has explored that being a student and 
young is associated with a high risk of addictive use of social media [10]. Addictive use of social media is associated with both neuroticism traits and depression [11,12]. Therefore, in this study, we aim to provide insight into how, and under what conditions, neuroticism leads to a higher level of depressive symptoms from the perspective of the addictive use of social media.

\subsection{The Relationship between Neuroticism and Depressive Symptoms}

The "Big Five" personality model (openness, conscientiousness, extraversion, agreeableness, neuroticism), as the most influential personality theoretical model in the world, has been widely studied by many psychological researchers in the past half-century and has been proved to have the relative consistency and stability across different samples, raters, languages, and cultures [13]. The personality trait of neuroticism refers to relatively stable tendencies to respond with negative emotions to threat, frustration, or loss [14]. Negative emotionality is the underpinning of neuroticism [15,16]. Individual neuroticism levels lead to differences in perception, memory, affection, and behavior during emotional stimuli and negative events, which explains why some individuals may be better at regulating their emotional state than others [17]. Neuroticism is significantly correlated with higher scores in depressive symptoms and appears to be the most powerful predictor of depression $[18,19]$.

\subsection{The Relationship between Addictive Use of Social Media and Depressive Symptoms}

With the development of computers and networks, people's communication mode has gradually shifted from reality to networks. According to the Pew Research Center, $72 \%$ of American adults use social media as of February 2019, including 90\% of young people aged 18-29 [20]. By March 2020, the number of Internet users in China has reached 904 million, of which $99.3 \%$ are mobile Internet users. In terms of occupation, student users account for $26.9 \%$ for the most substantial proportion. Among all kinds of social applications, WeChat has the highest usage rate, reaching 85.1\% [21]. Some studies have indicated a positive relationship between social media and mental health [22,23]. However, many others hold the opposite opinion, which shows that psychological distress is related to maladaptive use of both the Internet and the mobile phone [24-26]. Addictive use of social media (similar concepts include "social media use disorder", "social network overuse", "pathological social network use", "social network addiction") has a negative impact on adolescents' physical and mental development [27]. Overuse of Facebook can lead to depression, according to a report from the American Academy of Pediatrics [28]. Many studies have indicated that adolescents' problematic social network usage is positively correlated with depressive symptoms $[29,30]$. Therefore, the addictive use of social media of first-year students is worthy of attention.

\subsection{Addictive Use of Social Media as a Mediator in the Relationship between Neuroticism Trait and Depressive Symptoms}

Furthermore, previous studies showed that Facebook addiction was positively related to neuroticism [31,32]. Individuals with high neuroticism tend to use social media more frequently [33]. Introverted and neurotic people locate their "real me" on the Internet, while extroverts and non-neurotic people locate their "real me" through traditional social interaction [34]. Young adults high in neuroticism are inclined to present their ideal and false self on Facebook to a greater extent [35]. High-neurotic individuals may be more willing to seek emotional satisfaction and stress release through mobile phones to avoid the real world [36]. Therefore, we speculate that first-year college students with high neuroticism are more likely to be addicted to social media in the face of adaptive pressure, which will further aggravate their risk of depression. In other words, the addictive use of social media plays a mediating role between neuroticism and depressive symptoms.

\subsection{Moderation of Psychological Resilience}

Human responses to stress and trauma vary widely. Resilience is an interactive concept that refers to a relative resistance to environmental risk experiences or the overcoming of stress or adversity [37]. 
Psychological resilience is a protective factor of individuals in time of stress [38] and can increase the odds of not being depressed [39]. Among all independent variables, resilience is the most effective at predicting first-year university students' ability to adjust to university life [40]. At the same time, psychological resilience is a critical factor in protecting adolescents from internet addiction [41]. However, considering that neuroticism is the risk factor of individuals' addictive use of social media, while psychological resilience may be the protective factor for addictive use of social media, combined with the theory of the buffering effect of psychological resilience [42], this study assumes that psychological resilience may play a moderating role between neuroticism and addictive use of social media, namely psychological resilience may reduce the risk of addictive use of social media of highly neurotic individuals.

To sum up, based on the relationship between neuroticism and depressive symptoms, this study hypothesized a moderated mediation model (see Figure 1). We aimed to provide insights into how (the mediation role of addictive use of social media), and under what conditions (the moderator role of psychological resilience), neuroticism leads to a higher level of depressive symptoms.

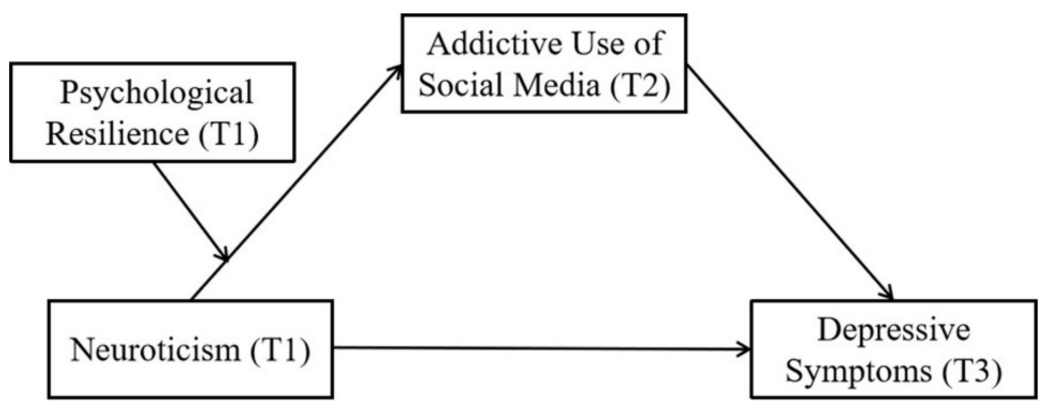

Figure 1. Moderated mediation model of the present research.

\section{Materials and Methods}

\subsection{Participants and Procedures}

Data were collected at three different periods among 1428 students who began their freshman year at a university in China, in 2017. The majors of these students include Law, Financial Management, Accounting, Electronic Information Engineering, Journalism, Advertising, Chinese Language and Literature, Marketing, Logistics Management, Economics, Network Engineering, English Language and Literature, Industrial Engineering, Business Administration, International Economics and Trade, Finance, Computer Science and Technology, and Electrical Engineering and Automation. The participation was anonymous and voluntary, based on the invitation from a course teacher responsible for the mental health education of this university. The teacher conducted the survey in each class on the spot with the participants' informed consent. The study protocol was approved by the institutional review board: Ethics Committee of Institute of Psychology, Chinese Academy of Sciences. Three waves of data were collected within two months after enrollment, considering that the first semester or even the first few weeks of college may be especially crucial in terms of student adaptation [43]. In wave 1 (October 2017), a total of 1428 students' demographic variables (T1), neuroticism (T1), psychological resilience (T1) and depressive symptoms (T1) were tested, and 1350 valid questionnaires were recovered, with an effective recovery rate of $94.54 \%$. In wave 2 (November 2017), the data of 1423 first-year students' addictive use of social media (T2) were collected, and 1262 valid questionnaires were recovered, with an effective recovery rate of $88.69 \%$. In wave 3 (December 2017), 1425 first-year students' depressive symptoms (T3) were measured, and 1271 valid questionnaires were recovered, with an effective recovery rate of $89.19 \%$.

Finally, the data of 1128 participants who were all valid in the three waves were included in the analysis, after eliminating the samples with obvious regularity, a large number of blank answers 
or those who only participated in one or two surveys. The range of participants's age was 17-22 years old $(\mathrm{M}=18.74 ; \mathrm{SD}=0.48)$. Among them, $731(64.80 \%)$ were female, $323(28.63 \%)$ were the only child in their family, and $309(27.40 \%)$ had their family in the city where the university is located.

\subsection{Measurement}

\subsubsection{Neuroticism}

Neuroticism was measured by the neuroticism subscale of the Ten-Item Personality Inventory (TIPI) [44], which contains two 7-point Likert items ("Anxious, easily upset." and "Calm, emotionally stable."; 1 = Disagree strongly, $7=$ Agree strongly). The second item above was scored in reverse. The correlation between neuroticism in the TIPI scale and that in BFI-44 is 0.81 [44]. The Chinese version of TIPI has good psychometric qualities in the Chinese sample [45].

\subsubsection{Depressive Symptoms}

The Patient Health Questionnaire (PHQ-9) [46] was used to assess the severity of depressive symptoms. The scale contains nine 4 -point Likert items $(0=$ Not at all, $3=$ Nearly every day $)$. An example of the items is "Over the last two weeks, how often have you been bothered by any of the following problems? Little interest or pleasure in doing things". In the present study, we use ten as the cut-off score $[47,48]$. Students with a total score of not lower than ten on PHQ-9 were judged as moderate to severe depressive symptoms severity. The Chinese version of PHQ-9 held good psychometric qualities in the Chinese sample [49]. Cronbach's alpha was 0.84 in this study.

\subsubsection{Addictive Use of Social Media}

The measurement of addictive use of social media referred to the FB addiction dimension in the Psycho-Social Aspect of Facebook Use Scale (PSAFU) [50], which includes four 5-point Likert items ( 1 = completely inconsistent; 5 = completely consistent). By replacing "Facebook" with "SNSs," we revised the scale to be more suitable for Chinese college students. An example of the items is "Some people from my surrounding have told me that I spend too much time on the SNSs." In this study, Cronbach's alpha was 0.69 .

\subsubsection{Psychological Resilience}

Psychological resilience was measured by the 10-item Connor-Davidson Resilience Scale (10-item CD-RISC) [51], which contains ten 5-point Likert items ( $1=$ never; $5=$ always). An example of the items is "Able to adapt to change." The higher the average score, the higher the psychological resilience level of the participants. The Chinese version of the 10-item CD-RISC held good psychometric properties and is applicable for Chinese people [52]. In this study, Cronbach's alpha was 0.90.

\subsection{Analytical Strategy}

PROCESS macro for SPSS with bootstrapping (95\% CI, 5000 samples) was used to test the moderated mediation model [53]. Controlled variables from wave 1 were added as covariates in the model, including gender $(1=$ Male, $0=$ Female $)$, age, the only child or not in the family $(1=$ Yes, $0=\mathrm{No})$, location of the family $(1=$ In the city where the university is located, $0=$ Outside the city where the university is located) and the baseline level of depressive symptoms.

\section{Results}

\subsection{Prevalence of Moderate to Severe Severity of Depressive Symptoms}

Among the 1128 students, the prevalence of moderate to severe severity of depressive symptoms was $10.28 \%$ in the first survey. There were 35 cases with moderate to severe severity of depressive symptoms (8.82\%) among 397 male students and 81 (11.08\%) among 731 female students. There was 
no statistically significant difference in the prevalence of moderate to severe severity of depressive symptoms between male and female students $\left(\chi^{2}=1.43, p=0.232\right)$ in the first survey.

In the third survey, the prevalence of moderate to severe severity of depressive symptoms was $11.17 \%$ among the 1128 students. There were 47 cases with moderate to severe severity of depressive symptoms (11.84\%) among 397 male students and 79 (10.81\%) among 731 female students. There was no statistically significant difference in the prevalence of moderate to severe severity of depressive symptoms between male and female students $\left(\chi^{2}=0.276, p=0.599\right)$ in the third survey.

Eighty-three students (7.36\%) who did not have moderate to severe depressive symptoms in the first survey turned to have in the third survey. On the other hand, 73 students of the total 1128 students $(6.47 \%)$ who had moderate to severe depressive symptoms in the first survey turned not to have in the third survey.

\subsection{Descriptive Statistics and Correlation Analysis}

Descriptive statistics and correlation analysis results were shown in Table 1. Neuroticism (T1) was positively correlated with depressive symptoms (T1). Addictive use of social media (T2) and depressive symptoms (T3), addictive use of social media (T2) was positively correlated with depressive symptoms (T1) and depressive symptoms (T3). Psychological resilience was negatively correlated with neuroticism (T1), depressive symptoms (T1), addictive use of social media (T2) and depressive symptoms (T3).

Table 1. Means, standard deviations, and correlations among research variables.

\begin{tabular}{|c|c|c|c|c|c|c|c|c|c|c|}
\hline $\begin{array}{l}\text { Research } \\
\text { Variables }\end{array}$ & $\mathbf{M}$ & SD & 1 & 2 & 3 & 4 & 5 & 6 & 7 & 8 \\
\hline \multicolumn{11}{|l|}{$\begin{array}{c}\text { 1.Gender } \\
(\text { male }=1 \\
\text { female }=0)\end{array}$} \\
\hline 2.Age & 18.74 & 0.83 & -0.02 & & & & & & & \\
\hline $\begin{array}{l}\text { 3.The Only } \\
\text { Child or Not }\end{array}$ & & & $0.239^{* * *}$ & $-0.134^{* * *}$ & & & & & & \\
\hline $\begin{array}{l}\text { 4.Location of } \\
\text { Family }\end{array}$ & & & -0.045 & 0.016 & -0.032 & & & & & \\
\hline $\begin{array}{l}\text { 5.Depressive } \\
\text { symptoms } \\
\text { (T1) }\end{array}$ & 0.62 & 0.38 & $-0.080^{* *}$ & 0.011 & $-0.066^{*}$ & 0.043 & & & & \\
\hline $\begin{array}{l}\text { 6.Neuroticism } \\
\text { (T1) }\end{array}$ & 3.60 & 1.12 & $-0.111^{* * *}$ & -0.012 & -0.029 & -0.02 & $0.411^{* * *}$ & & & \\
\hline $\begin{array}{l}\text { 7.Psychological } \\
\text { Resilience (T1) }\end{array}$ & 3.51 & 0.61 & $0.180^{* * *}$ & 0.041 & 0.05 & -0.022 & $-0.355^{* * *}$ & $-0.415^{* * *}$ & & \\
\hline $\begin{array}{l}\text { 8. Addictive } \\
\text { Use of Social } \\
\text { Media (T2) }\end{array}$ & 2.28 & 0.74 & -0.014 & 0.009 & -0.001 & -0.022 & $0.214^{* * *}$ & $0.177^{* * *}$ & $-0.148^{* * *}$ & \\
\hline $\begin{array}{l}\text { 9.Depressive } \\
\text { symptoms } \\
\text { (T3) }\end{array}$ & 0.67 & 0.39 & -0.022 & -0.04 & $-0.059 *$ & 0.004 & $0.507^{* * *}$ & $0.270^{* * *}$ & $-0.239 * * *$ & $0.300^{* * *}$ \\
\hline
\end{tabular}

Note: ${ }^{*} p<0.05 ;{ }^{* *} p<0.01 ; * * * p<0.01$.

\subsection{Relationship Between Neuroticism and Sepressive Symptoms: Test of a Moderated Mediation Model}

The results of polynomial regression analysis were shown in Table 2. After controlling for gender, age, the only child or not in family, location of family, and depressive symptoms level (T1), neuroticism (T1) positively predicted depressive symptoms (T3) $(B=0.03, S E=0.01, t=2.71, p=0.007)$ significantly. Neuroticism (T1) had a significant positive effect on levels of addictive use of social media (T2) $(B=0.05, S E=0.02, t=2.34, p=0.02)$. Addictive use of social media (T2) positively predicted depressive symptoms (T3) $(B=0.10, S E=0.01, t=7.64, p<0.001)$ significantly. After controlling for addictive use of social media (T2), neuroticism (T1) still positively predicted depressive symptoms 
(T3) $(B=0.02, S E=0.01, t=2, p=0.045)$ significantly. The Bootstrap test using Model 4 of PROCESS showed that addictive use of social media (T2) played a significant mediating role between neuroticism (T1) and depressive symptoms (T3) $(95 \%$ Boot CI $=(0.002,0.013))$.

Table 2. Multiple regression results of the moderated mediation model.

\begin{tabular}{|c|c|c|c|c|c|c|}
\hline Independent Variable & $B$ & $S E$ & $t$ & $p$ & $R^{2}$ & $F$ \\
\hline \multicolumn{7}{|c|}{ Dependent Variable: Depressive symptoms (T3) } \\
\hline Gender & 0.03 & 0.02 & 1.23 & 0.221 & \multirow{6}{*}{0.27} & \multirow{6}{*}{$67.57^{* * *}$} \\
\hline Age & -0.02 & 0.01 & -1.92 & 0.056 & & \\
\hline The Only Child or Not & -0.03 & 0.02 & -1.53 & 0.127 & & \\
\hline Location of Family & -0.01 & 0.02 & -0.53 & 0.599 & & \\
\hline Depressive symptoms (T1) & 0.48 & 0.03 & 16.91 & $<0.001$ & & \\
\hline Neuroticism (T1) & 0.03 & 0.01 & 2.71 & 0.007 & & \\
\hline \multicolumn{7}{|c|}{ Dependent Variable: Addictive Use of Social Media (T2) } \\
\hline Gender & 0.03 & 0.05 & 0.66 & 0.509 & \multirow{8}{*}{0.07} & \multirow{8}{*}{$10.55^{* * *}$} \\
\hline Age & 0.01 & 0.03 & 0.26 & 0.791 & & \\
\hline The Only Child or Not & 0.02 & 0.05 & 0.39 & 0.700 & & \\
\hline Location of Family & -0.04 & 0.05 & -0.92 & 0.358 & & \\
\hline Depressive symptoms (T1) & 0.33 & 0.06 & 5.27 & $<0.001$ & & \\
\hline Neuroticism (T1) & 0.05 & 0.02 & 2.34 & 0.020 & & \\
\hline Psychological Resilience (T1) & -0.08 & 0.04 & -1.99 & 0.047 & & \\
\hline $\begin{array}{c}\text { Neuroticism }(\mathrm{T} 1) \times \\
\text { Psychological Resilience }(\mathrm{T} 1)\end{array}$ & 0.10 & 0.03 & 3.63 & $<0.001$ & & \\
\hline \multicolumn{7}{|c|}{ Dependent Variable: Depressive symptoms (T3) } \\
\hline Gender & 0.03 & 0.02 & 1.20 & 0.230 & \multirow{7}{*}{0.30} & \multirow{7}{*}{$69.21^{* * *}$} \\
\hline Age & -0.02 & 0.01 & -2.05 & 0.041 & & \\
\hline The Only Child or Not & -0.04 & 0.02 & -1.66 & 0.098 & & \\
\hline Location of Family & -0.01 & 0.02 & -0.33 & 0.741 & & \\
\hline Depressive symptoms (T1) & 0.45 & 0.03 & 15.89 & $<0.001$ & & \\
\hline Neuroticism (T1) & 0.02 & 0.01 & 2.00 & 0.045 & & \\
\hline $\begin{array}{l}\text { Addictive Use of Social } \\
\text { media (T2) }\end{array}$ & 0.10 & 0.01 & 7.64 & $<0.001$ & & \\
\hline
\end{tabular}

Note: ${ }^{* * *} p<0.001 ; B$ - unstandardized regression weight; SE-standard error for the unstandardized regression weight; $t$ - $t$-test statistic; $F-F$-test statistic.

To examine the moderation effect of psychological resilience (T1) in this mediation model, we applied Model 7 in PROCESS macro to do the Bootstrap test. Neuroticism (T1) and psychological resilience (T1) were centralized before constructing the interaction term. The results showed that the mediating effect was significantly moderated by psychological resilience (T1), indicating that the moderated mediation model was established $(95 \%$ Boot $C I=(0.003,0.019))$. Specifically, the mediating effect was not significant at low level of psychological resilience $(-1$ SD; $95 \%$ Boot CI $=(-0.008,0.006))$, while was significant at medium level $(95 \%$ Boot $C I=(0.000,0.011))$ and high level of psychological resilience $(+1 \mathrm{SD} ; 95 \%$ Boot $\mathrm{CI}=(0.005,0.019))$. The mediating effect of addictive use of social media (T2) at different levels of psychological resilience (T1) was shown in Figure 2. 


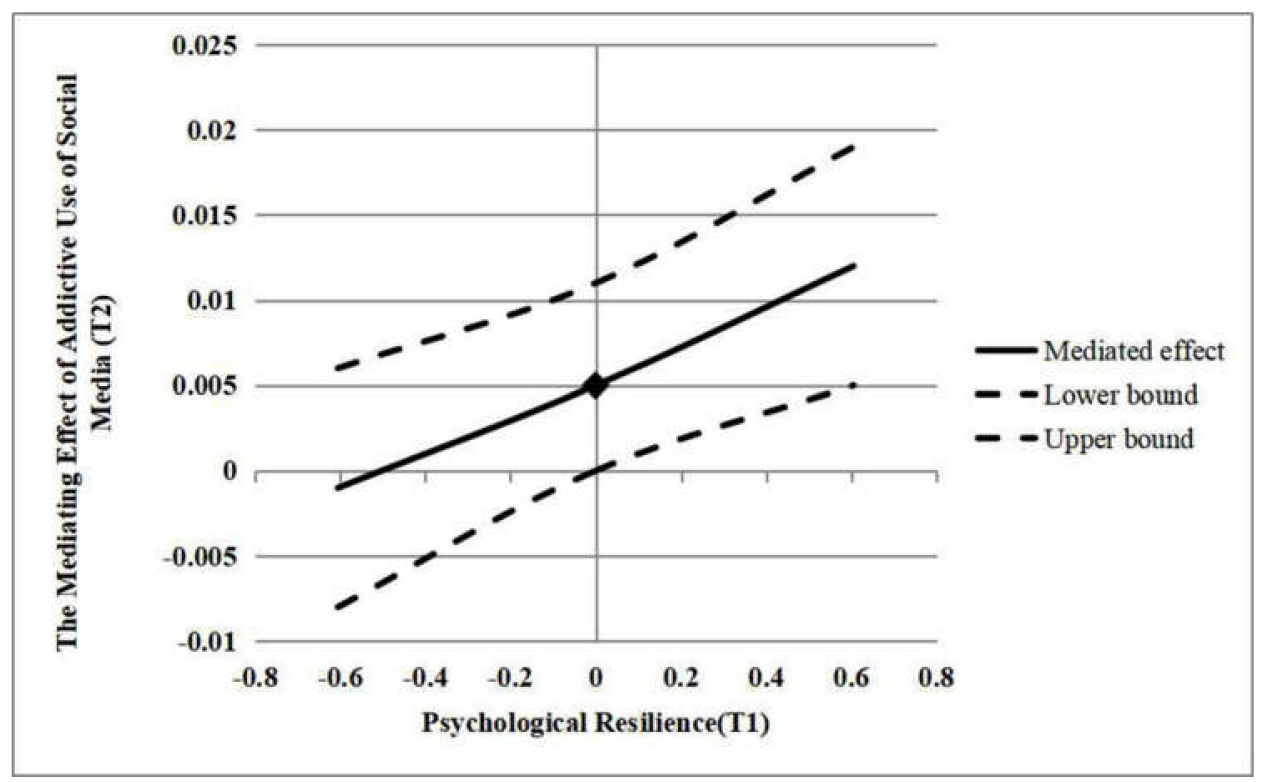

Figure 2. The mediating effect of addictive use of social media (T2) at different levels of psychological resilience $(\mathrm{T} 1)$.

Meanwhile, the neuroticism $(\mathrm{T} 1) \times$ psychological resilience $(\mathrm{T} 1)$ interaction significantly predicted addictive use of social media (T2) $(B=0.10, S E=0.03, t=3.63, p<0.001$; see Figure 3$)$. The influence of neuroticism (T1) on addictive use of social media (T2) was not significant when the level of psychological resilience (T1) is low ( $B=-0.01, S E=0.03, t=-0.31, p=0.757)$ and was significant when psychological resilience (T1) is at medium $(B=0.05, S E=0.02, t=2.34, p=0.02)$ and high level $(B=0.11, S E=0.03$, $t=4.23, p<0.001)$.

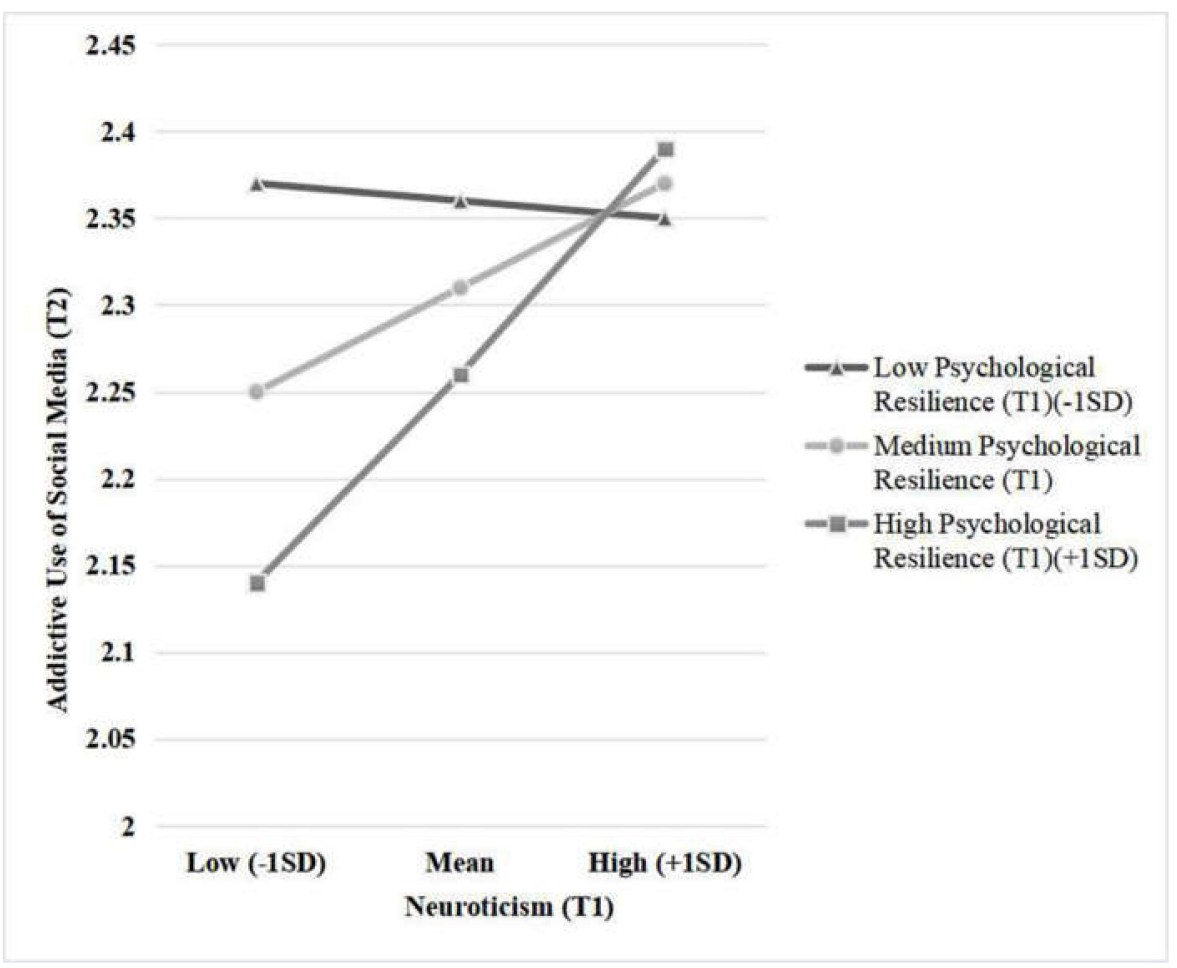

Figure 3. Simple slope test of the interaction between neuroticism (T1) and psychological resilience (T1) on the addictive use of social media (T2). 


\section{Discussion}

In this study, the relationship between neuroticism and depressive symptoms of first-year students was investigated by longitudinal tracking. The mediating and moderating effects of addictive use of social media and psychological resilience were explored, and a moderated mediation model was constructed. The results help to understand the two critical issues of how and under which conditions the neuroticism of first-year college students affect their depressive symptoms.

\subsection{Prevalence of Moderate to Severe Severity of Depressive Symptoms}

Among the 1128 students, the prevalence of moderate to severe severity of depressive symptoms was $10.28 \%$ in the first survey, and $11.17 \%$ in the third survey with the cut-off score no less than 10 of PHQ-9 scores. Of the 397 male students, 35 cases had moderate to severe severity of depressive symptoms $(8.82 \%)$ in the first survey and $47(11.84 \%)$ in the third survey. Of the 731 female students, 81 cases had moderate to severe severity of depressive symptoms (11.08\%) in the first survey and 79 $(10.81 \%)$ in the third survey. Moreover, 83 students $(7.36 \%)$ who did not have moderate to severe severity of depressive symptoms when they enrolled in the college turned to do two months later. However, previous studies showed that the prevalence of depression under the same standard ranged from $4.23 \%$ to $7.00 \%$ in the general Chinese population [ 49,54$]$, which is $9.56 \%$ in males and $5.60 \%$ in females [49]. The results suggest that first-year students have a higher prevalence of moderate to severe severity of depressive symptoms than that in the general population, representing it is necessary to pay attention to and research the adaptation problems and depressive symptoms of first-year students, considering the higher risk of depression for the students with poor school adjustment [3].

\subsection{Mediation of Addictive Use of Social Media}

This study shows that first-year college students' neuroticism can significantly predict depressive symptoms two months later. High neuroticism people usually show more emotion dysregulation and psychological inflexibility, which can also explain why individuals with high neuroticism are more prone to depression [9]. This study also found that neuroticism can significantly predict the addictive use of social media, indicating that the higher the level of neuroticism, the more likely an addiction to social media, which is consistent with the previous research results [33,55]. Meanwhile, the addictive use of social media can significantly predict depressive symptoms. The higher the intensity of first-year students' addictive use of social media, the higher the risk of depression, which is in line with previous researches $[28,29,56]$.

This study confirmed that the addictive use of social media plays a significant mediating role in the influencing process of neuroticism on depressive symptoms. This paper further explains the mechanism of neuroticism's influence on depressive symptoms; that is, neuroticism affects the depressive symptoms level of first-year students directly and indirectly through addictive use of social media, which serves as a bridge between neuroticism and depressive symptoms.

\subsection{Moderation of Psychological Resilience}

Psychological resilience plays a moderating role in the relationship between neuroticism and the addictive use of social media. The improvement of psychological resilience does not reduce the risk of addictive social media use of high neurotic individuals. However, it protects the low neuroticism ones. Namely, with the improvement of psychological resilience, low neuroticism individuals reduce their addictive use of social media significantly. This also suggests that in addition to the buffering effect (reducing the risk of high-risk individuals), there is also an increasing effect (making low-risk individuals perform better) of psychological resilience, which echoes the current research results [57]. Our results show that low neurotic individuals likewise have a risk of addictive use of social media if their psychological resilience level is not high. 
At the same time, this study has constructed a moderated mediation model to investigate the moderating role of resilience in the mediating process of "neuroticism -> addictive use of social media -> depressive symptoms". The results show that neuroticism directly affects the depressive symptoms level of individuals with low resilience. With the improvement of resilience, neuroticism impacts the level of depressive symptoms through the mediating effect of addictive use of social media. Therefore, first-year college students with high psychological resilience, regardless of their neuroticism, can avoid depression and promote their adaptation to college life from the perspective of intervention in the addictive use of social media. However, this approach is not appropriate with low resilience, and other interventions should be sought. For example, when both neuroticism and resilience are low, the latter can be improved by the optimal level of participation in sports activities according to gender and age [58].

\section{Implication and Limitation}

\subsection{Implication}

Theoretically, the present study implied that low neuroticism people with low psychological resilience might also have a risk of addictive use of social media. Second, psychological resilience acts not as a buffer, but as an increasing effect, making people with low neuroticism less likely to be addicted to social media. Third, the mediating effect of addictive use of social media is different in people with different psychological resilience. That is, the mediating effect was not significant at the low level of psychological resilience, but was significant at the medium and high level of psychological resilience. These results provide a more sophisticated knowledge of understanding the depressive symptoms of first-year college students.

Practically, the present study provides insights into how and under what conditions, neuroticism leads to a higher level of depressive symptoms, which enables several practical implications for reducing the risk of depression of first-year college students with adaptation problems: (a) after controlling the baseline level of depressive symptoms, neuroticism and addictive use of social media are all risk factors of first-year college students' depression. Therefore, research on the personality traits of first-year college students and dynamic evaluation of their social media behaviors can be undergone after enrollment to predict their depressive symptoms two months after effectively. (b) More accurate classification guidance can be offered based on differences in first-year college students' personality characteristics and resilience. On the one hand, the possibility of the addictive use of social media of low neuroticism individuals can be reduced by improving their psychological resilience according to the moderating effect. On the other hand, based on the results of the mediating effect, individuals with high resilience need attention to avoid the addictive use of social media to better adapt to college life.

\subsection{Limitation}

Although some valuable results have been obtained in this study, there are still some deficiencies. First, neuroticism in this study involves a relatively broad personality construct without subdividing different aspects. In future research, the neuroticism personality dimension can be subdivided to distinguish the impact of different aspects on the relationship between addictive use of social media and depressive symptoms. Second, this study used longitudinal data to analyze and provided evidence to support the hypothesized path from the addictive use of social media to depressive symptoms. However, some studies pointed out that depression was a critical antecedent variable of social media addiction $[59,60]$. Future research can start from this perspective to explore the more abundant dyadic relationship between the two.

\section{Conclusions}

The current research focuses on the depressive symptoms of first-year college students. It aims to provide insights into how (the mediation role of addictive use of social media), and under what 
conditions (the moderator role of psychological resilience), neuroticism leads to a higher level of depressive symptoms. The most obvious findings to emerge from this study are that low neuroticism people with low psychological resilience might also have a risk of addictive use of social media, psychological resilience acts as an increasing effect decreasing the risk of low neuroticism individual's addictive use of social media, and addictive use of social media plays the mediating effect only with medium and high psychological resilience. The insights gained from this study may help understand the depressive symptoms of first-year college students and provide intervention guidance for the adaptation problems.

Author Contributions: Conceptualization, M.Z., W.M., D.Z., Y.W. and F.L.; methodology, W.M., D.Z., F.L. and M.Z.; formal analysis, W.M. and D.Z.; investigation, Y.W., F.L. and L.Y.; resources, Y.W., L.Y. and K.W.; data curation, L.Y. and Y.W.; writing-original draft preparation, D.Z. and W.M.; writing-review and editing, M.Z., W.M. and D.Z.; visualization, D.Z., W.M. and K.W.; supervision, M.Z.; project administration, M.Z. All authors have read and agreed to the published version of the manuscript.

Funding: This research received no external funding.

Acknowledgments: We wish to thank the students who participated in this research on a voluntary basis.

Conflicts of Interest: The authors declare no conflict of interest.

\section{References}

1. Leong, F.T.L.; Bonz, M.H.; Zachar, P. Coping styles as predictors of college adjustment among freshmen. Couns. Psychol. Q. 1997, 10, 211-220. [CrossRef]

2. Dyson, R.; Renk, K. Freshmen adaptation to university life: Depressive symptoms, stress, and coping. J. Clin. Psychol. 2006, 62, 1231-1244. [CrossRef] [PubMed]

3. Chui, R.C.-F.; Chan, C.-K. School Adjustment, Social Support, and Mental Health of Mainland Chinese College Students in Hong Kong. J. Coll. Stud. Dev. 2017, 58, 88-100. [CrossRef]

4. Garlow, S.J.; Rosenberg, J.; Moore, J.D.; Haas, A.P.; Koestner, B.; Hendin, H.; Nemeroff, C.B. Depression, desperation, and suicidal ideation in college students: Results from the American Foundation for Suicide Prevention College Screening Project at Emory University. Depress. Anxiety 2008, 25, 482-488. [CrossRef] [PubMed]

5. Jasso-Medrano, J.L.; López, F. Measuring the relationship between social media use and addictive behavior and depression and suicide ideation among university students. Comput. Hum. Behav. 2018, 87, $183-191$. [CrossRef]

6. Martin, M. Neuroticism as predisposition toward depression: A cognitive mechanism. Pers. Individ. Differ. 1985, 6, 353-365. [CrossRef]

7. Saklofske, D.; Kelly, I.; Janzen, B. Neuroticism, depression, and depression proneness. Pers. Individ. Differ. 1995, 18, 27-31. [CrossRef]

8. Klein, D.N.; Kotov, R.; Bufferd, S.J. Personality and depression: Explanatory models and review of the evidence. Annu. Rev. Clin. Psychol. 2011, 7, 269-295. [CrossRef]

9. Paulus, D.J.; Vanwoerden, S.; Norton, P.J.; Sharp, C. Emotion dysregulation, psychological inflexibility, and shame as explanatory factors between neuroticism and depression. J. Affect. Disord. 2016, 190, 376-385. [CrossRef]

10. Andreassen, C.S.; Pallesen, S.; Griffiths, M.D. The relationship between addictive use of social media, narcissism, and self-esteem: Findings from a large national survey. Addict. Behav. 2017, 64, 287-293. [CrossRef]

11. Gao, T.; Xiang, Y.-T.; Zhang, H.; Zhang, Z.; Mei, S. Neuroticism and quality of life: Multiple mediating effects of smartphone addiction and depression. Psychiatry Res. Neuroimaging 2017, 258, 457-461. [CrossRef] [PubMed]

12. Turel, O.; Poppa, N.T.; Gil-Or, O. Neuroticism Magnifies the Detrimental Association between Social Media Addiction Symptoms and Wellbeing in Women, but Not in Men: A three-Way Moderation Model. Psychiatr. Q. 2018, 89, 605-619. [CrossRef] [PubMed] 
13. John, O.P.; Naumann, L.P.; Soto, C.J. Paradigm shift to the integrative big five trait taxonomy: History, measurement, and conceptual issues. In Handbook of Personality: Theory and Research; John, O.P., Robins, R.W., Pervin, L.A., Eds.; Guilford Press: New York, NY, USA, 2008; pp. 114-158. [CrossRef]

14. Lahey, B.B. Public health significance of neuroticism. Am. Psychol. 2009, 64, 241-256. [CrossRef] [PubMed]

15. Digman, J.M. Higher-order factors of the Big Five. J. Pers. Soc. Psychol. 1997, 73, 1246-1256. [CrossRef]

16. Otani, K.; Suzuki, A.; Matsumoto, Y.; Shirata, T.; Noto, K.; Kanno, M. Implication of core beliefs about negative-self in neuroticism. Int. J. Psychiatry Clin. Pract. 2020, 1-6. [CrossRef]

17. Canli, T. Toward a Neurogenetic Theory of Neuroticism. Ann. N. Y. Acad. Sci. 2008, 1129, 153-174. [CrossRef]

18. Enns, M.W.; Cox, B.J. Personality dimensions and depression: Review and commentary. Can. J. Psychiatry 1997, 42, 274-284. [CrossRef]

19. Pereira-Morales, A.; Adan, A.; Forero, D.A. Perceived Stress as a Mediator of the Relationship between Neuroticism and Depression and Anxiety Symptoms. Curr. Psychol. 2017, 38, 66-74. [CrossRef]

20. Social Media Fact Sheet. Available online: http://www.pewinternet.org/fact-sheet/social-media (accessed on 24 June 2020).

21. The 45th Statistical Report on Internet Development in China. Available online: http://www.cnnic.net.cn/ hlwfzyj/hlwxzbg/hlwtjbg/202004/t20200428_70974.htm (accessed on 24 June 2020).

22. Kim, J.-H.; Lee, J.-E.R. The Facebook Paths to Happiness: Effects of the Number of Facebook Friends and Self-Presentation on Subjective Well-Being. Cyberpsychol. Behav. Soc. Netw. 2011, 14, 359-364. [CrossRef]

23. Valkenburg, P.M.; Peter, J.; Schouten, A.P. Friend Networking Sites and Their Relationship to Adolescents' Well-Being and Social Self-Esteem. CyberPsychol. Behav. 2006, 9, 584-590. [CrossRef]

24. Beranuy, M.; Oberst, U.; Carbonell, X.; Chamarro, A.; Chamarro, A. Problematic Internet and mobile phone use and clinical symptoms in college students: The role of emotional intelligence. Comput. Hum. Behav. 2009, 25, 1182-1187. [CrossRef]

25. Twenge, J.M.; Joiner, T.E.; Rogers, M.L.; Martin, G.N. Increases in Depressive Symptoms, Suicide-Related Outcomes, and Suicide Rates Among U.S. Adolescents After 2010 and Links to Increased New Media Screen Time. Clin. Psychol. Sci. 2017, 6, 3-17. [CrossRef]

26. Vannucci, A.; Flannery, K.M.; Ohannessian, C.M. Social media use and anxiety in emerging adults. J. Affect. Disord. 2017, 207, 163-166. [CrossRef] [PubMed]

27. Woods, H.C.; Scott, H. \#Sleepyteens: Social media use in adolescence is associated with poor sleep quality, anxiety, depression and low self-esteem. J. Adolesc. 2016, 51, 41-49. [CrossRef]

28. O'Keeffe, G.S.; Clarke-Pearson, K. The Impact of Social Media on Children, Adolescents, and Families. Pediatrics 2011, 127, 800-804. [CrossRef]

29. Kelly, Y.; Zilanawala, A.; Booker, C.; Sacker, A. Social Media Use and Adolescent Mental Health: Findings From the UK Millennium Cohort Study. EClinicalMedicine 2019, 6, 59-68. [CrossRef]

30. Giota, K.G.; Kleftaras, G. The role of personality and depression in problematic use of social networking sites in Greece. Cyberpsychology J. Psychosoc. Res. Cyberspace 2013, 7. [CrossRef]

31. Andreassen, C.S.; Torsheim, T.; Brunborg, G.S.; Pallesen, S. Development of a Facebook Addiction Scale. Psychol. Rep. 2012, 110, 501-517. [CrossRef]

32. Marengo, D.; Poletti, I.; Settanni, M. The interplay between neuroticism, extraversion, and social media addiction in young adult Facebook users: Testing the mediating role of online activity using objective data. Addict. Behav. 2020, 102, 106150. [CrossRef]

33. Correa, T.; Hinsley, A.W.; Gil De Zúñiga, H. Who interacts on the Web?: The intersection of users' personality and social media use. Comput. Hum. Behav. 2010, 26, 247-253. [CrossRef]

34. Amichai-Hamburger, Y.; Wainapel, G.; Fox, S. “On the Internet No One Knows I'm an Introvert”: Extroversion, Neuroticism, and Internet Interaction. CyberPsychology Behav. 2002, 5, 125-128. [CrossRef] [PubMed]

35. Michikyan, M.; Subrahmanyam, K.; Dennis, J. Can you tell who I am? Neuroticism, extraversion, and online self-presentation among young adults. Comput. Hum. Behav. 2014, 33, 179-183. [CrossRef]

36. Ho, S.S.; Lwin, M.O.; Lee, E.W. Till logout do us part? Comparison of factors predicting excessive social network sites use and addiction between Singaporean adolescents and adults. Comput. Hum. Behav. 2017, 75, 632-642. [CrossRef]

37. Rutter, M. Implications of Resilience Concepts for Scientific Understanding. Ann. N. Y. Acad. Sci. 2006, 1094, 1-12. [CrossRef] 
38. Ong, A.D.; Bergeman, C.S.; Bisconti, T.L.; Wallace, K.A. Psychological resilience, positive emotions, and successful adaptation to stress in later life. J. Pers. Soc. Psychol. 2006, 91, 730-749. [CrossRef]

39. Edward, K.-L. Resilience: A Protector From Depression. J. Am. Psychiatr. Nurses Assoc. 2005, 11, $241-243$. [CrossRef]

40. Rahat, E.; Ilhan, T. Coping Styles, Social Support, Relational Self-Construal, and Resilience in Predicting Students' Adjustment to University Life. Educ. Sci. Theory Pr. 2016, 16, 187-208. [CrossRef]

41. Wisniewski, P.; Jia, H.; Wang, N.; Zheng, S.; Xu, H.; Rosson, M.B.; Carroll, J.M. Resilience Mitigates the Negative Effects of Adolescent Internet Addiction and Online Risk Exposure. In Proceedings of the 33rd Annual ACM Conference on Human Factors in Computing Systems, Seoul, Korea, 2015; Association for Computing Machinery: New York, NY, USA, 2015; pp. 4029-4038.

42. Sheerin, C.M.; Lind, M.J.; Brown, E.A.; Gardner, C.O.; Kendler, K.S.; Amstadter, A.B. The impact of resilience and subsequent stressful life events on MDD and GAD. Depression Anxiety 2017, 35, 140-147. [CrossRef]

43. Bowman, N.A.; Jarratt, L.; Jang, N.; Bono, T.J. The Unfolding of Student Adjustment During the First Semester of College. Res. High. Educ. 2018, 60, 273-292. [CrossRef]

44. Gosling, S.D.; Rentfrow, P.J.; Swann, W.B., Jr. A very brief measure of the Big-Five personality domains. J. Res. Pers. 2003, 37, 504-528. [CrossRef]

45. Li, J. Psychometric Properties of Ten-Item Personality Inventory in China. China J. Health Psychol. 2013, 21, 1689-1692. [CrossRef]

46. Kroenke, K.; Spitzer, R.L. The PHQ-9: A New Depression Measure. Psychiatr. Ann. 2002, 32, 509-515. [CrossRef]

47. Min, B.-q.; Zhou, A.-h.; Liang, F.; Jia, J. Clinical application of Patient Health Questionnaire for self-administered measurement (PHQ-9) as screening tool for depression. J. Neurosci. Ment. Health 2013, 13, 569-572. [CrossRef]

48. Manea, L.; Gilbody, S.; McMillan, D. Optimal cut-off score for diagnosing depression with the Patient Health Questionnaire (PHQ-9): A meta-analysis. Can. Med. Assoc. J. 2011, 184, E191-E196. [CrossRef] [PubMed]

49. Wang, W.; Bian, Q.; Zhao, Y.; Li, X.; Wang, W.; Du, J.; Zhang, G.; Zhou, Q.; Zhao, M. Reliability and validity of the Chinese version of the Patient Health Questionnaire (PHQ-9) in the general population. Gen. Hosp. Psychiatry 2014, 36, 539-544. [CrossRef] [PubMed]

50. Bodroža, B.; Jovanovic, T. Validation of the new scale for measuring behaviors of Facebook users: Psycho-Social Aspects of Facebook Use (PSAFU). Comput. Hum. Behav. 2016, 54, 425-435. [CrossRef]

51. Campbell-Sills, L.; Stein, M.B. Psychometric analysis and refinement of the connor-davidson resilience scale (CD-RISC): Validation of a 10-item measure of resilience. J. Trauma. Stress 2007, 20, 1019-1028. [CrossRef]

52. Wang, L.; Shi, Z.; Zhang, Y.; Zhang, Z. Psychometric properties of the 10-item Connor-Davidson Resilience Scale in Chinese earthquake victims. Psychiatry Clin. Neurosci. 2010, 64, 499-504. [CrossRef]

53. Hayes, A.F. Introduction to Mediation, Moderation, and Conditional Process. Analysis: A Regression-Based Approach; Guilford Publications: New York, NY, USA, 2013.

54. Yu, N.X.; Tam, W.W.S.; Wong, P.T.; Lam, T.H.; Stewart, S.M. The Patient Health Questionnaire-9 for measuring depressive symptoms among the general population in Hong Kong. Compr. Psychiatry 2012, 53, 95-102. [CrossRef]

55. Cho, H.-Y.; Kim, D.J.; Park, J.W. Stress and adult smartphone addiction: Mediation by self-control, neuroticism, and extraversion. Stress Health 2017, 33, 624-630. [CrossRef]

56. Donnelly, E.; Kuss, D.J. Depression among Users of Social Networking Sites (SNSs): The Role of SNS Addiction and Increased Usage. J. Addict. Prev. Med. 2016, 2, 107. [CrossRef]

57. Zhang, J.; Zhou, M.; Yu, N.X. Neighborhood Characteristics and Older Adults' Well-Being: The Roles of Sense of Community and Personal Resilience. Soc. Indic. Res. 2017, 137, 1490. [CrossRef]

58. Griciūtè, A. Optimal Level of Participation in Sport Activities According to Gender and Age can be Associated with Higher Resilience: Study of Lithuanian Adolescents. Sch. Ment. Health 2015, 8, 257-267. [CrossRef] 
59. Chang, F.-C.; Chiu, C.-H.; Lee, C.-M.; Chen, P.-H.; Miao, N.-F. Predictors of the initiation and persistence of Internet addiction among adolescents in Taiwan. Addict. Behav. 2014, 39, 1434-1440. [CrossRef]

60. Li, G.; Hou, G.; Yang, N.; Jian, H.; Wang, W. Relationship between anxiety, depression, sex, obesity, and internet addiction in Chinese adolescents: A short-term longitudinal study. Addict. Behav. 2019, 90, 421-427. [CrossRef] 